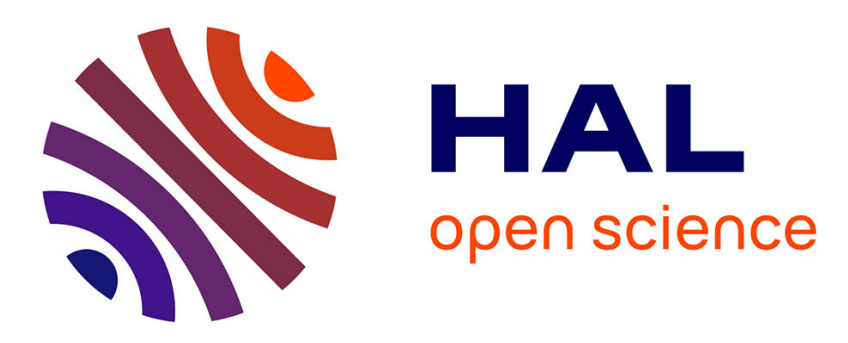

\title{
Numerical scheme for a stratigraphic model with erosion constraint and nonlinear gravity flux
}

Clément Cancès, Didier Granjeon, Nicolas Peton, Quang Huy Tran, Sylvie

Wolf

\section{- To cite this version:}

Clément Cancès, Didier Granjeon, Nicolas Peton, Quang Huy Tran, Sylvie Wolf. Numerical scheme for a stratigraphic model with erosion constraint and nonlinear gravity flux. FVCA 8 - 2017 - International Conference on Finite Volumes for Complex Applications VIII, Jun 2017, Lille, France. pp.327-335, 10.1007/978-3-319-57394-6_35. hal-01639681

\section{HAL Id: hal-01639681 \\ https://hal.science/hal-01639681}

Submitted on 20 Nov 2017

HAL is a multi-disciplinary open access archive for the deposit and dissemination of scientific research documents, whether they are published or not. The documents may come from teaching and research institutions in France or abroad, or from public or private research centers.
L'archive ouverte pluridisciplinaire HAL, est destinée au dépôt et à la diffusion de documents scientifiques de niveau recherche, publiés ou non, émanant des établissements d'enseignement et de recherche français ou étrangers, des laboratoires publics ou privés. 


\title{
Numerical scheme for a stratigraphic model with erosion constraint and nonlinear gravity flux
}

\author{
Clément Cancès, Didier Granjeon, Nicolas Peton, Quang Huy Tran, \\ and Sylvie Wolf
}

\begin{abstract}
In this work, we study an extension of the model introduced by Eymard et al. [Int. J. Numer. Methods Engrg. 60, 527-248 (2004)] for the simulation of large scale transport processes of sediments, subject to an erosion constraint. The novelty we consider lies in the diffusion law relating the flux of sediments and the slope of the topography, that now involves a $p$-Laplacian with $p>2$ in order to get more realistic landscape evolutions. This physical sophistication entails the construction of an entirely new numerical scheme, the details of which shall be supplied.
\end{abstract}

Key words: Stratigraphic forward modeling, gravity-driven sediment transport, weather-limited erosion, evolutionary $p$-Laplacian, complementarity problem

MSC (2010): 35K85, 65M08, 86-08

\section{A constrained model arising in stratigraphic modeling}

We are interested in the evolution of the sediment height $h: \Omega \times \mathbb{R}_{+}$where $\Omega=$ $\left(0, L_{x}\right) \times\left(0, L_{y}\right) \subset \mathbb{R}^{2}$ is a rectangular computational domain, the sea level being fixed to $h=0$. The sediments are transported from the top to the bottom, due to gravity. The "natural" sediment flux $\mathbf{F}: \Omega \times(0, T) \rightarrow \mathbb{R}^{2}$ is given by

$$
\mathbf{F}=-K(h)|\nabla h|^{p-2} \nabla h=-|\nabla h|^{p-2} \nabla \psi(h),
$$

Clément Cancès

Team RAPSODI, Inria Lille - Nord Europe,

40 avenue Halley, 59650 Villeneuve d'Ascq, France

e-mail: clement.cances@inria.fr

Didier Granjeon, Nicolas Peton, Quang Huy Tran, and Sylvie Wolf

IFP Energies nouvelles, 1 \& 4 avenue de Bois Préau, 92852 Rueil-Malmaison Cedex, France

e-mail: \{didier.granjeon, nicolas.peton, quang-huy.tran, sylvie.wolf $\} @$ ifpen.fr 
where the diffusion coefficient takes the form $K(h)=K_{c}$ if $h \geq 0$ and $K(h)=K_{m}$ if $h \leq 0$ ( $c$ stands for continental and $m$ for maritime), and where $\psi(h)=\int_{0}^{h} K(a) \mathrm{d} a$. It is known by geomorphologists that sedimentation $\left(\partial_{t} h \geq 0\right)$ and erosion $\left(\partial_{t} h<0\right)$ processes are non-symmetric: soil material must first be produced in situ by weathering processes prior to being transported by diffusion. As a consequence, it is postulated that the erosion is limited from below by $-E$, where the quantity $E>0$ corresponds to a known maximal production rate of sediments, i.e.,

$$
\partial_{t} h+E \geq 0 \quad \text { in } \Omega \times \mathbb{R}_{+} .
$$

In order to incorporate this constraint in the problem, we follow the approach of [3] that consists in introducing a multiplier $\lambda: \Omega \times \mathbb{R}_{+} \rightarrow[0,1]$ to reduce the flux in a conservative way. More precisely, we impose that

$$
\begin{aligned}
\partial_{t} h+\nabla \cdot(\lambda \mathbf{F})=0 & \text { in } \Omega \times \mathbb{R}_{+}, \\
(1-\lambda)\left(\partial_{t} h+E\right)=0 & \text { in } \Omega \times \mathbb{R}_{+},
\end{aligned}
$$

where (3b) expresses that locally either the erosion constraint is saturated $\left(\partial_{t} h=\right.$ $-E$ ) or the flux is unlimited $(\lambda=1)$. Combining the inequality (2), the reduction assumption $\lambda \leq 1$ with (3), we end up with the synthetic system

$$
\begin{aligned}
\partial_{t} h+\nabla \cdot(\lambda \mathbf{F})=0, & \text { in } \Omega \times \mathbb{R}_{+}, \\
\min \{1-\lambda, \gamma[E-\nabla \cdot(\lambda \mathbf{F})]\}=0 & \text { in } \Omega \times \mathbb{R}_{+},
\end{aligned}
$$

in which $\mathbf{F}$ is given by (1) and in which the complementarity equation (4b) involves a scaling parameter $\gamma>0$ whose role is to make the two arguments of the min function homogeneous. We impose the inflow of sediment across the boundary, i.e.,

$$
\mathbf{F} \cdot \mathbf{n}=\phi \leq 0 \quad \text { on } \partial \Omega \times \mathbb{R}_{+}
$$

where $\mathbf{n}$ is the outward normal to $\partial \Omega$. Finally, we prescribe the initial condition

$$
h_{\mid t=0}=h^{0} \quad \text { in } \Omega, \quad \text { with } \quad h_{\star} \leq h^{0} \leq h^{\star}
$$

for some $h_{\star}, h^{\star} \in \mathbb{R}$. The goal of this contribution is to propose a numerical scheme to approximate the solutions $(h, \lambda)$ of $(1),(4)-(6)$.

In comparison with the previous contributions [3-5], here our attention is restricted to the case of a single lithology but we lay emphasis on the nonlinearity $p>2$ in the definition (1) of the flux $\mathbf{F}$. The reason why such a nonlinearity should be incorporated into the model comes from the experimental observation the "linear" diffusion law $\mathbf{F}=-\nabla \psi(h)$ do not hold for most sedimentary systems of interest. In particular, the linear $(p=2)$ gravity flux implies that sediment propagation occurs at infinite speed. Thus, one of the motivation for considering $p>2$ is to recover propagation at finite speeds, which in turn enable geologists to track down knickpoints. 
Fig. 1: The original Cartesian grid (red dots at center) is surrounded by ghost cells (dashed blue) to be used to impose the boundary conditions. We distinguish lateral ghost cells (green dots at center) and corner ghost cells (blue dots at center). The dual cells (shaded yellow) admit the primal cell centers as vertices. Concerning the edges, the inner edges $\mathscr{E}_{\text {int }}$ (solid black) and the boundary edges $\mathscr{E}_{\text {ext }}$ (solid green) are treated in a different way.

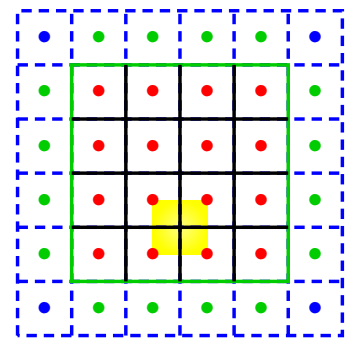

\section{A cell-centered discretization on Cartesian grids}

Roughly speaking, the problem to be solved numerically consists of a constrained evolutionary $p$-Laplacian, complemented with Neumann boundary conditions. In order to discretize the natural fluxes (1), we use a semi-explicit Finite Volume scheme inspired from [1].

Let $N_{x}, N_{y}$ be two positive integers, then $\Omega=\left(0, L_{x}\right) \times\left(0, L_{y}\right)$ is discretized into inner cells $C_{i, j}=((i-1) \Delta x, i \Delta x) \times((j-1) \Delta y, j \Delta y)$ where $\Delta x=L_{x} / N_{x}$ and $\Delta y=$ $L_{y} / N_{y}$. The center of $C_{i, j}$ is denoted by $\mathbf{x}_{i, j}=((i-1 / 2) \Delta x,(j-1 / 2) \Delta y)$. In order to impose the boundary condition (5), we extend the grid with ghost cells. Let

$$
\begin{aligned}
\mathscr{L} & =\left\{1, \ldots, N_{x}\right\} \times\left\{0, N_{y}+1\right\} \cup\left\{0, N_{x}+1\right\} \times\left\{1, \ldots, N_{y}\right\} \\
\mathscr{I} & =\left\{1, \ldots, N_{x}\right\} \times\left\{1, \ldots, N_{y}\right\}
\end{aligned}
$$

be respectively the set of ghost cells (green dots in Fig. 1) and that of inner primal cells. The set of the edges between the primal cells is denoted by $\mathscr{E}$. Two particular subsets of $\mathscr{E}$ will be used in the sequel: the subset $\mathscr{E}_{\text {int }}$ of the inner edges (between two inner cells) and the subset $\mathscr{E}_{\text {ext }}$ of the boundary edges (between an inner cell and a ghost cell), as depicted in Fig. 1. Time is discretized by $0=t_{0}<t_{1}<\ldots<t_{n}<\ldots$, in which the time-step is denoted by $\Delta t_{n}=t_{n+1}-t_{n}$.

The primal unknowns $\left(h_{i, j}^{n}, \lambda_{i, j}^{n}\right)$, for $(i, j) \in \mathscr{I}$ and $n \geq 1$, are located at the centers of the inner cells and of the lateral ghost cells (cf. Fig. 1). The initial data $h^{0}$ is discretized into a piecewise-constant function. For $(i, j) \in \mathscr{I}$, we set

$$
h_{i, j}^{0}=\frac{1}{\Delta x \Delta y} \int_{C_{i, j}} h^{0}(\mathbf{x}) \mathrm{d} \mathbf{x},
$$

while for $(i, j) \in \mathscr{L}$, a simple extrapolation is used to obtain $h_{i, j}^{0}$. To approximate the unconstrained flux $\mathbf{F} \cdot \mathbf{n}$, we first approximate $|\nabla h|^{p-2}$ on the dual cells by

$$
\begin{aligned}
B_{i+1 / 2, j+1 / 2}^{n}= & \left\{\frac{1}{2}\left(\frac{h_{i+1, j}^{n}-h_{i, j}^{n}}{\Delta x}\right)^{2}+\frac{1}{2}\left(\frac{h_{i+1, j+1}^{n}-h_{i, j+1}^{n}}{\Delta x}\right)^{2}\right. \\
& \left.+\frac{1}{2}\left(\frac{h_{i, j+1}^{n}-h_{i, j}^{n}}{\Delta y}\right)^{2}+\frac{1}{2}\left(\frac{h_{i+1, j+1}^{n}-h_{i+1, j}^{n}}{\Delta y}\right)^{2}\right\}^{p / 2-1},
\end{aligned}
$$


which can be seen as an approximation of $|\nabla h|^{2}$ raised to the power $p / 2-1$. It is worth noting that this approximation for $|\nabla h|^{2}$ is coercive: it cannot vanish unless the four values on the dual cell are identical. The unconstrained flux $\mathbf{F} \cdot \mathbf{n}$ across the inner edges of $\mathscr{E}_{\mathrm{int}}$ at time $t_{n+1}$ is then computed thanks to the semi-implicit formulae

$$
\begin{aligned}
& F_{i+1 / 2, j}^{n+1}=\frac{B_{i+1 / 2, j-1 / 2}^{n}+B_{i+1 / 2, j+1 / 2}^{n}}{2} \cdot \frac{\psi\left(h_{i, j}^{n+1}\right)-\psi\left(h_{i+1, j}^{n+1}\right)}{\Delta x}, \\
& F_{i, j+1 / 2}^{n+1}=\frac{B_{i-1 / 2, j+1 / 2}^{n}+B_{i+1 / 2, j+1 / 2}^{n}}{2} \cdot \frac{\psi\left(h_{i, j}^{n+1}\right)-\psi\left(h_{i, j+1}^{n+1}\right)}{\Delta y},
\end{aligned}
$$

whereas the boundary fluxes are prescribed by (5), that is,

$$
F_{1 / 2, j}^{n+1}=-\frac{1}{\Delta t_{n} \Delta y} \int_{t_{n}}^{t_{n+1}} \int_{(j-1) \Delta y}^{j \Delta y} \phi(x=0, y) \mathrm{d} y \mathrm{~d} t
$$

and similar relations for $F_{N_{x}+1 / 2, j}^{n+1}, F_{i, 1 / 2}^{n+1}$ and $F_{i, N_{y}+1 / 2}^{n+1}$. For $(i, j) \in \mathscr{I}$ and $n \geq 0$, the first equation of (4) is discretized into

$$
\frac{h_{i, j}^{n+1}-h_{i, j}^{n}}{\Delta t_{n}}+D_{i, j}^{n+1}=0,
$$

where the discrete divergence

$$
D_{i, j}^{n+1}=\frac{(\lambda F)_{i+1 / 2, j}^{n+1}-(\lambda F)_{i-1 / 2, j}^{n+1}}{\Delta x}+\frac{(\lambda F)_{i, j+1 / 2}^{n+1}-(\lambda F)_{i, j-1 / 2}^{n+1}}{\Delta y}
$$

involves the upwinded flux

$$
\begin{aligned}
& (\lambda F)_{i+1 / 2, j}^{n+1}=\lambda_{i, j}^{n+1}\left(F_{i+1 / 2, j}^{n+1}\right)^{+}-\lambda_{i+1, j}^{n+1}\left(F_{i+1 / 2, j}^{n+1}\right)^{-}, \\
& (\lambda F)_{i, j+1 / 2}^{n+1}=\lambda_{i, j}^{n+1}\left(F_{i, j+1 / 2}^{n+1}\right)^{+}-\lambda_{i, j+1}^{n+1}\left(F_{i, j+1 / 2}^{n+1}\right)^{-},
\end{aligned}
$$

in which $a^{+}=\max \{a, 0\}$ and $a^{-}=-\min \{a, 0\}$ are the positive and negative parts of the real number $a$. The boundary fluxes are not limited, so we impose that $\lambda_{i, j}^{n+1}=1$ for $(i, j) \in \mathscr{L}$. As for the complementarity equation (4b), it is discretized by

$$
\min \left\{1-\lambda_{i, j}^{n+1}, \frac{\Delta x \Delta y}{\langle F\rangle_{i, j}^{n+1}}\left[E-D_{i, j}^{n+1}\right]\right\}=0
$$

where $\langle F\rangle_{i, j}^{n+1}=\Delta y\left[\left(F_{i+1 / 2, j}^{n+1}\right)^{+}+\left(F_{i-1 / 2, j}^{n+1}\right)^{-}\right]+\Delta x\left[\left(F_{i, j+1 / 2}^{n+1}\right)^{+}+\left(F_{i, j-1 / 2}^{n+1}\right)^{-}\right]$represents the total unlimited outgoing flux from cell $(i, j)$. The choice of the local weight $\gamma_{i, j}^{n+1}=\Delta x \Delta y /\langle F\rangle_{i, j}^{n+1}$ is justified by the following property.

Lemma 1. Equation (15) is equivalent to 


$$
\lambda_{i, j}^{n+1}=\min \left\{1, \frac{\Delta x \Delta y E+\rangle \lambda F\left\langle_{i, j}^{n+1}\right.}{\langle F\rangle_{i, j}^{n+1}}\right\}
$$

where $\rangle \lambda F\left\langle_{i, j}^{n+1}=\Delta y\left[\lambda_{i-1, j}^{n+1}\left(F_{i-1 / 2, j}^{n+1}\right)^{+}+\lambda_{i+1, j}^{n+1}\left(F_{i+1 / 2, j}^{n+1}\right)^{-}\right]+\Delta x\left[\lambda_{i, j-1}^{n+1}\left(F_{i, j-1 / 2}^{n+1}\right)^{+}+\right.\right.$ $\left.\lambda_{i, j+1}^{n+1}\left(F_{i, j+1 / 2}^{n+1}\right)^{-}\right]$is the total limited incoming flux into cell $(i, j)$. This implies, in particular, that

$$
0<\lambda_{i, j}^{n+1} \leq 1
$$

Proof. The discrete divergence (13)-(14) can be easily transformed into

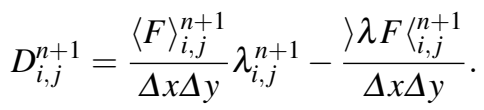

Multiplication by $-\gamma_{i, j}^{n+1}$ makes $-\lambda_{i, j}^{n+1}$ appear alone in the second argument of the min in (15). As a result, we can extract $-\lambda_{i, j}^{n+1}$ out of the min to obtain (16). We infer from (16) that $\lambda_{i, j}^{n+1} \leq 1$, and from (15) that $D_{i, j}^{n+1} \leq E$. It follows from (18) that $E \Delta x \Delta y+\rangle \lambda F\left\langle_{i, j}^{n+1} \geq\langle F\rangle_{i, j}^{n+1} \Delta x \Delta y \geq 0\right.$. From (16), we infer that $\lambda_{i, j}^{n+1}>0$.

Contrary to [3-5], we advocate mounting the whole system (12)-(14), (16) in the unknowns $\left(h_{i, j}^{n+1}, \lambda_{i, j}^{n+1}\right)$. This avoids the task of switching variables according to whether or not the constraint is saturated.

Lemma 2. For all $n \geq 0$, one has

$$
\begin{aligned}
\min _{(i, j) \in \mathscr{I}} h_{i, j}^{n} & \geq h_{\star}, \\
\sum_{(i, j) \in \mathscr{I}} h_{i, j}^{n} \Delta x \Delta y & =\int_{\Omega} h^{0} \mathrm{~d} \mathbf{x}-\int_{0}^{t_{n}} \int_{\partial \Omega} \phi \mathrm{d} \gamma \mathrm{d} t .
\end{aligned}
$$

Proof. The above estimates rely on induction. The mass balance (19b) is obtained by summing (12) over $(i, j) \in \mathscr{I}$. To derive (19a), let $\left(i_{\star}, j_{\star}\right) \in \mathscr{I}$ such that $h_{i_{\star}, j_{\star}}^{n+1}=$ $\min _{(i, j) \in \mathscr{I}} h_{i, j}^{n+1}$. Then, $D_{i_{\star}, j_{\star}}^{n+1} \leq 0$, hence $h_{i_{\star}, j_{\star}}^{n+1} \geq h_{i_{\star}, j_{\star}}^{n} \geq h_{\star}$.

As a consequence of Lemma 2 , one gets a $L_{\text {loc }}^{\infty}\left(\mathbb{R}_{+} ; L^{1}(\Omega)\right)$ estimate on the discrete sediment height, namely,

$$
\sum_{i} \sum_{j}\left|h_{i, j}^{n}\right| \Delta x \Delta y \leq 2 h_{\star}^{-}|\Omega|+\int_{\Omega} h^{0} \mathrm{~d} \mathbf{x}-\int_{0}^{t_{n}} \int_{\partial \Omega} \phi \mathrm{d} \gamma \mathrm{d} t .
$$

From this and thanks to a topological degree argument [2], we can prove that the scheme admits at least one solution, as claimed in the following Proposition.

Proposition 1. Let $h_{i, j}^{n},(i, j) \in \mathscr{I}$, be such that (19) hold. Then, for all $\Delta t_{n}>0$, there exists at least one solution $\left(h_{i, j}^{n+1}, \lambda_{i, j}^{n+1}\right)$ to the nonlinear system (12)-(15) satisfying (17)-(19). 


\section{Numerical results}

In order to illustrate the capabilities of the model and the numerical scheme, we show two test cases. We consider a basin of size $180 \mathrm{~km} \times 180 \mathrm{~km}$ where the topography represents a continental domain made of mountains $\left(K_{c}=500 \mathrm{~km}^{2} / \mathrm{My}\right)$ and a marine domain $\left(K_{m}=10 \mathrm{~km}^{2} / \mathrm{My}\right)$. The sea level is $h=0 \mathrm{~km}$. Two incoming fluxes constant in time are prescribed on the left and right borders as

$$
-\int_{0}^{T} \int_{0}^{L_{y}} \phi(0, y, t) \mathrm{d} y \mathrm{~d} t=24.0 T \mathrm{~km}^{2},-\int_{0}^{T} \int_{0}^{L_{y}} \phi\left(L_{x}, y, t\right) \mathrm{d} y \mathrm{~d} t=26.6 T \mathrm{~km}^{2} .
$$

To see the influence of the constraint upon the erosion rate, we consider no flux limitation in the first case (Fig. 2). To this end, we take $E \gg 1 \mathrm{~km} / \mathrm{My}$, such that $\lambda$ keeps the constant value 1 in the domain. In the second test case (Figs.3-4), we activate the constraint by taking $E=0.04 \mathrm{~km} / \mathrm{My}$.

Numerically, the domain is made up of $361 \times 361$ cells. The exponent for the $p$-Laplacian in (1) is set at $p=2.5$. Simulations are run until $T=1 \mathrm{My}$. At each time step, the nonlinear system is solved within a threshold of $10^{-5} \mathrm{~km}$ by Newton's method. The linear system arising at each Newton iteration is solved by means of PETSc routines and using the BiCGSTAB method with the ILU(0) preconditonner. The time steps vary dynamically as follows. We start with $\Delta t_{0}=10^{-4} \mathrm{My}$. If the time iteration is accepted, we set $\Delta t_{n+1}=1.1 \Delta t_{n}$ subject to the (commonly accepted) maximum value $\Delta t_{n+1} \leq 10^{-3}$ My. If Newton's method fails after 10 iterations, the time step is rejected and we restart the iteration with $\Delta t_{n}:=0.5 \Delta t_{n}$.
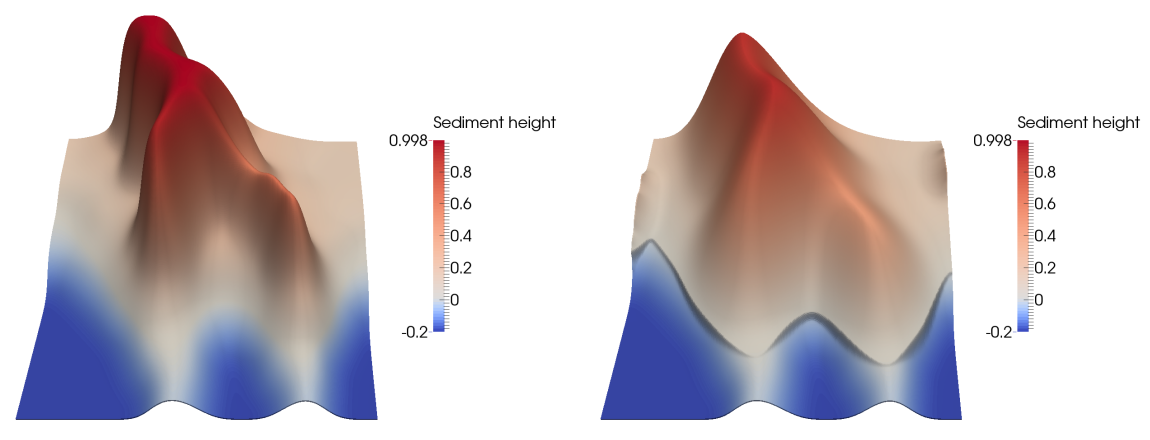

Fig. 2: Case without constraint: initial state (left) and final state (right) of $h(\mathrm{~km})$.

In the test case with no erosion constraint (Fig. 2), we observe that after $1 \mathrm{My}$ the diffusion has notably smoothed the global structure of the mountains, especially in steep areas. We can also distinguish the shoreline between the continental and the marine domains. This is due to the contrast between the diffusion coefficients $K_{c}$ and $K_{m}$. In Table 1, we summarize some numerical data associated with the simulation. We can see that this test case is an "easy" one as no time steps were refused. 


\begin{tabular}{|c|c|}
\hline Accepted time steps & 1016 \\
\hline Refused time steps & 0 \\
\hline Mean Newton iterations per accepted time step & 1.98 \\
\hline Mean solver iterations per Newton iteration & 1.99 \\
\hline Computing time (s) & 783 \\
\hline
\end{tabular}

Table 1: Numerical data for the case without constraint.

The mean number of required Newton iterations is rather low, as well as the mean number of solver iterations. However, larger values of the diffusion coefficients may cause more severe difficulties, implying much smaller time steps.
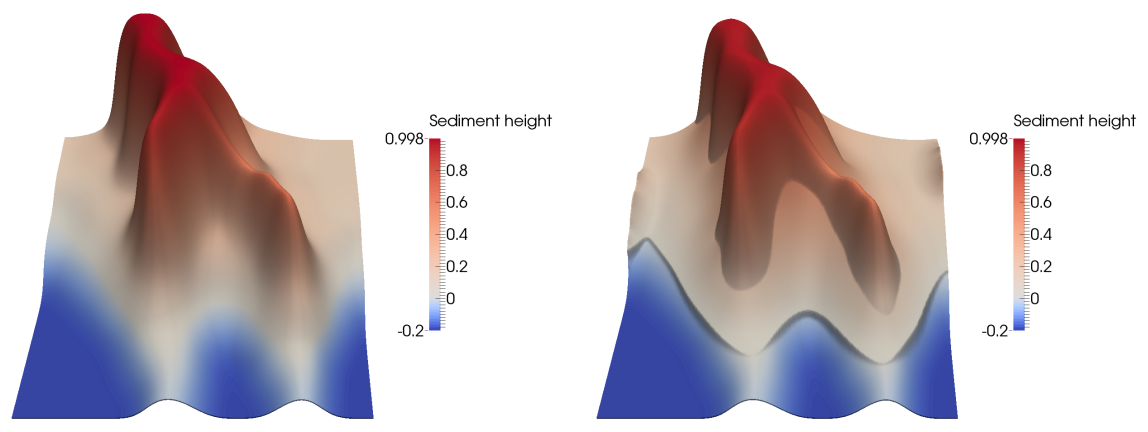

Fig. 3: Case with constraint: initial state (left) and final state (right) of $h(\mathrm{~km})$.
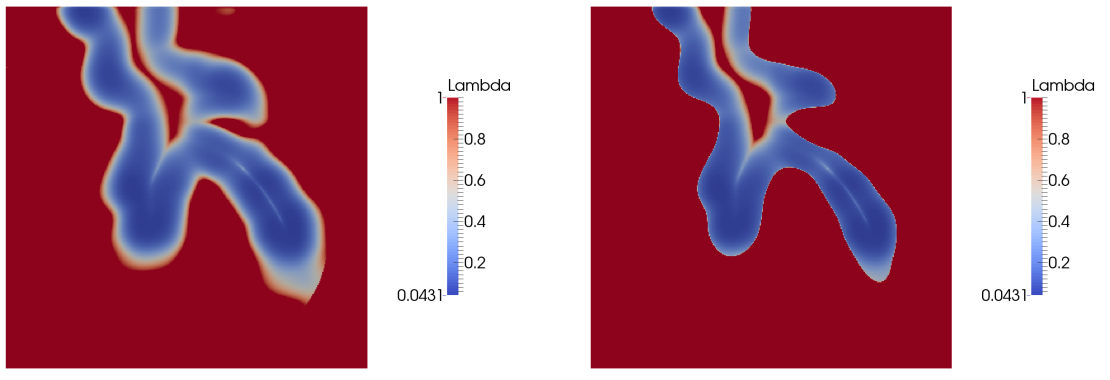

Fig. 4: Case with constraint: initial state (left) and final state (right) of $\lambda$.

In the second test case (Fig. 3) where the erosion constraint enters into play, we observe a different behavior. After $1 \mathrm{My}$, the mountains underwent less erosion and their structure is still recognizable. We can visualize the areas where the constraint is 


\begin{tabular}{|c|c|}
\hline Accepted time steps & 1235 \\
\hline Refused time steps & 95 \\
\hline Mean Newton iterations per accepted time step & 2.29 \\
\hline Mean solver iterations per Newton iteration & 12.14 \\
\hline Computing time (s) & 1809 \\
\hline
\end{tabular}

Table 2: Numerical results for the case with constraint.

effective by looking at the values of the flux limiter $\lambda$ in Fig. 4 . We can notice that $\lambda$ takes values close to zero in the areas corresponding to the mountain's flanks, where the diffusion is the most important. The shoreline between the continental and marine domains is still present, for the same reason as before. By looking at Table 2 we also notice some differences. With the same management of the time steps as in the previous case, Newton's method sometimes fails to converge and some time steps are rejected. The model is more difficult to solve numerically: the mean number of Newton iterations and mean number of solver iterations are higher than in the case without constraint. This accounts for the rise in the computing time between the two simulations. Furthermore, it has been observed that the cases without constraint and with a very strong constraint were relatively easy to compute. The difficulties are most serious for "intermediate" values of the maximum erosion rate $E$.

\section{Conclusion}

This extension of the model [3] to a $p$-Laplacian diffusion law is the first step of a broader program whose objective is to enrich the physics of the industrial simulator Dionisos Flow ${ }^{\mathrm{TM}}$, developed by IFP Energies nouvelles. The next steps include usual features such as multi-lithology and variable bathymetry, but also a coupling of the sediment flow with water effects such as rains and rivers.

\section{References}

1. Andreianov, B., Boyer, F., Hubert, F.: Finite volume schemes for the $p$-Laplacian on Cartesian meshes. M2AN Math. Model. Numer. Anal. 38(6), 931-959 (2004)

2. Eymard, R., Gallouët, T., Ghilani, M., Herbin, R.: Error estimates for the approximate solutions of a nonlinear hyperbolic equation given by finite volume schemes. IMA J. Numer. Anal. 18(4), 563-594 (1998)

3. Eymard, R., Gallouët, T., Granjeon, D., Masson, R., Tran, Q.H.: Multi-lithology stratigraphic model under maximum erosion rate constraint. Int. J. Numer. Methods Engrg. 60(2), 527-548 (2004)

4. Gervais, V.: Étude et simulation d'un modèle stratigraphique multi-lithologique sous contrainte de taux d'érosion maximal. Ph.D. thesis, Université de Provence, Aix-Marseille I (2004)

5. Gervais, V., Masson, R.: Numerical simulation of a stratigraphic model. Comput. Geosci. 12(2), 163-179 (2008) 